Int. J. Dev. Biol. 55: 65-72 (2011)

doi: $10.1387 / \mathrm{ijdb} .092970 \mathrm{da}$

\title{
Gene expression profiling identifies eleven DNA repair genes down-regulated during mouse neural crest cell migration
}

\author{
DOMENICO ALBINO ${ }^{1}$, ANTONELLA BRIZZOLARA ${ }^{2}$, STEFANO MORETTI ${ }^{3}$, CARLA FALUGI ${ }^{4}$, \\ VALENTINA MIRISOLA ${ }^{5}$, PAOLA SCARUFFI ${ }^{6}$, MICHELE DI CANDIA $^{5}$, MAURO TRUINI ${ }^{7}$, SIMONA COCO $^{6}$, \\ STEFANO BONASSI ${ }^{3}$ and GIAN PAOLO TONINI*,6

\begin{abstract}
${ }^{1}$ Laboratory of the Italian Neuroblastoma Foundation, ${ }^{2}$ Laboratory of Immunological Therapy, National Cancer Research Institute (IST), ${ }^{3}$ CNRS, Université Paris-Dauphine, LAMSADE, ${ }^{4}$ Department of Biology, University of Genoa, ${ }^{5}$ Functional Genomics, National Cancer Research Institute, Genoa, Italy (CNR-IEIIT, Genoa) ${ }^{6}$ Translational Oncopathology, National
\end{abstract} \\ Cancer Research Institute (IST) and ${ }^{7}$ Department of Pathology, National Cancer Research Institute, Genoa, Italy
}

\begin{abstract}
Neural Crest Cells (NCCs) are transient multipotent migratory cells that derive from the embryonic neural crest which is itself derived from the margin of the neural tube. DNA repair genes are expressed in the early stages of mammalian development to reduce possible replication errors and genotoxic damage. Some birth defects and cancers are due to inappropriate or defective DNA repair machinery, indicating that the proper functioning of DNA repair genes in the early stages of fetal development is essential for maintaining DNA integrity. We performed a genome-wide expression analysis combining laser capture microdissection (LCM) and highdensity oligo-microarray of murine NCCs at pre-migratory embryonic days 8.5 (E8.5), and at E13.5, as well as on neural crest-derived cells from the adrenal medulla at postnatal day 90 . We found 11 genes involved in DNA repair activity (response to DNA damage stimulus, DNA damage checkpoint, base-excision repair, mismatch repair), over-expressed in the early stages of mouse embryo development. Expression of these 11 genes was very low or undetectable in the differentiated adrenal medulla of the adult mouse. Amongst the 11 genes, 6 had not been previously reported as being over-expressed during mouse embryonic development. High expression of DNA repair genes in enriched NCCs during early embryonic development may contribute to maintaining DNA integrity whilst failure of some of these genes may be associated with the onset of genetic disease and cancer. Our model of enriched murine NCCs and neural crest-derived cells can be used to elucidate the key roles of genes during normal embryonic development and in cancer pathogenesis.
\end{abstract}

KEY WORDS: neural crest cell, laser capture microdissection, microarray, neuroblastoma

\section{Introduction}

In mammals the Neural Crest (NC) is an embryonic structure derived from the margin of the neural tube, which generates different cell and tissue types, including NCCs (Gammill and Bronner-Fraser, 2003). NCCs are a transient and intriguing multipotent migratory cell population, composed mainly of multipotent cells whose fate is determined before their migration by environmental factors and by pre-committed cells. NCCs can be classified according to the four functional domains of the NC from which they originate, namely, the cranial (cephalic) neural crest, the trunk neural crest (TNC), the vagal neural crest and the sacral neural crest. Trunk neural crest (TNC) cells migrate ventrolaterally through the anterior half of each sclerotome and give rise to the sympathetic and parasympathetic ganglia, the dorsal

\footnotetext{
Abbreviations used in this paper: AG, adrenal gland; AM, adrenal medulla; BER, base excision repair; DRG,dorsal root ganglia; EASE, expression analysis systematic explorer; HR, homologous recombination; LCM, laser capture microdissection; MMR, mismatch repair; NCCs, neural crest cells; NER, nucleotide excision repair; NT, neural tube; TNC, trunk neural crest; qRTPCR, quantitative real-time PCR; SAM, significance analysis of microarrays.
}

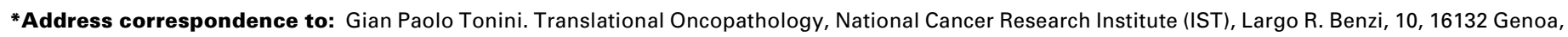
Italy. Fax: +39-010-573-7487. e-mail: gianpaolo.tonini@istge.it

Supplementary Material (two tables) for this paper is available at: http://dx.doi.org/10.1387/ijdb.092970da 
root ganglia (DRG) and adrenal medulla (AM) cells (Le Dourain and Kalcheim, 1999). The remaining TNC cells migrate dorsolaterally under the surface ectoderm and become pigment producing melanocytes. The complex patterns of gene expression associated with pre-migratory NCCs and post-migratory NCCderived cells can be used to obtain information about gene categories that are down- or up-regulated during development.

During embryonic life, NCCs differentiate and migrate in response to several environmental signals. The maintenance of genomic integrity is essential for their correct development. DNA damage caused by genotoxic agents (Frosina, 2000; Dusre et al. 1989), occurring after gamete formation and before postnatal life may impair genomic integrity and gene expression (Jaroudi and SenGupta, 2007). The DNA repair machinery is a complex system comprising several genes involved in diverse processes including error-prone repair, base-excision repair and mismatch repair. These processes are activated in response to DNA damage stimuli. Failure of one or more DNA repair pathways is a frequent catalyst for carcinogenesis.

The study of molecular crosstalk between different cell types within a tissue can be greatly facilitated by the use of laser capture microdissection (LCM). The LCM technique allows for the efficient isolation of single cells with no or very little contamination from

\section{TABLE 1}

\section{NUMBER OF GENES FOUND DIFFERENTIALLY EXPRESSED BY SAM ANALYSIS AND GENE CATEGORIES FOUND BY EASE ANALYSIS}

\begin{tabular}{lccc} 
A & Total & Up-regulated & Down-regulated \\
\hline E8.5 vs E13.5 & 498 & 209 & 289 \\
E13.5 vs P90 & 176 & 117 & 59 \\
\hline
\end{tabular}

\begin{tabular}{lcccccc}
\multirow{2}{*}{ System } & \multicolumn{2}{c}{ Up-regulated in E8.5 vs E13.5 } & & Down-regulated in E8.5 vs E13.5 \\
\cline { 2 - 3 } \cline { 5 - 6 } & Gene Category & EASE score & & Gene Category & EASE score \\
\hline GO Biological Process & DNA replication & $2.43 \mathrm{E}+07$ & & cell adhesion & $4.46 \mathrm{E}+09$ \\
GO Biological Process & cell proliferation & $6.02 \mathrm{E}+05$ & & cell communication & $1.15 \mathrm{E}-04$ \\
GO Biological Process & cell cycle & $3.36 \mathrm{E}+03$ & & neurogenesis & $3.61 \mathrm{E}-03$ \\
GO Biological Process & $\begin{array}{c}\text { cell growth and/or } \\
\text { maintenance }\end{array}$ & $8.47 \mathrm{E}-03$ & & cell-cell signaling & $5.69 \mathrm{E}-03$ \\
& & & & \\
\hline
\end{tabular}

\begin{tabular}{|c|c|c|c|c|}
\hline \multirow[b]{2}{*}{ System } & \multicolumn{2}{|c|}{ Up-regulated in E13.5 vs P90 } & \multicolumn{2}{|c|}{ Down-regulated in E13.5 vs P90 } \\
\hline & Gene Category & EASE score & Gene Category & EASE score \\
\hline GO Biological Process & development & $5.32 \mathrm{E}+09$ & $\begin{array}{c}\text { steroid } \\
\text { biosynthesis }\end{array}$ & $9.06 \mathrm{E}-04$ \\
\hline GO Biological Process & neurogenesis & $6.38 \mathrm{E}+09$ & hormone biosynthesis & $1.05 \mathrm{E}-03$ \\
\hline GO Biological Process & $\begin{array}{l}\text { microtubule-based } \\
\text { process }\end{array}$ & $1.62 \mathrm{E}-04$ & lipid biosynthesis & $1.32 \mathrm{E}-02$ \\
\hline GO Biological Process & organogenesis & $1.11 \mathrm{E}-02$ & $\begin{array}{c}\text { monocarboxylic acid } \\
\text { transport }\end{array}$ & 1.16E-02 \\
\hline
\end{tabular}

The table shows the total number of genes differentially expressed between pre-migratory E8.5 and post-migratory E13.5 (developmental stages) and between post-migratory E13.5 and adult P90 AM (3 months old mouse) using SAM analysis (A). Stage-to-stage comparison was performed to identify the most relevant genes up-regulated and down-regulated in enriched NCCs and NCC-derived cells during mouse development. Lists of differentially expressed genes were loaded into EASE (http://david.niaid.nih.gov/david/ease.htm) and only gene categories with an EASE score higher than 0.05 are reported here. Moreover, only GO Biological Process results from EASE were considered, excluding GO Cellular Component and GO Molecular Function. Functional categories into which genes found differentially expressed by SAM analysis for E8.5 versus E13.5 stage comparisons (B) and for E13.5 versus adult P90 AM stage comparisons (C), are shown. surrounding tissue components, simultaneously leaving the intracellular structure and molecules intact (Hergenhahn M, 2003).

In this study, we utilized LCM and high-density oligonucleotide microarrays to identify genes involved in DNA repair mechanisms in embryonic and adult mice. We analyzed the gene expression profiles of pre-migratory enriched NCCs during the early stages of development and of post-migratory differentiated NCC-derived cells. In this way, we identified a number of novel DNA repair genes that were highly expressed in early developmental stages. The efficacy of the experimental approach was confirmed by the concomitant identification of previously reported DNA repair genes.

Data providing a comprehensive expression profile of DNA repair genes during the early stages of embryonic development are currently incomplete due to the limited number of available cells. The hypothesis that mammalian NCCs exhibit higher DNA repair activity than differentiated cells is supported in the literature (Jaroudi and SenGupta, 2007; Vinson and Hales, 2002; Tichy and Stambrook, 2008; Saretzki etal.2004). In fact, failure of DNA repair genes is associated with several syndromes and malignances that arise in the perinatal age: neuroblastomas, Wilms tumor, embryonic rhabdomyosarcoma, medulloblastomas, medulloepithelioma, ependymoblastoma, medullomyoblastoma (MacDonald, 2008).

Previously, the expression of DNA repair genes in mouse embryonic NCCs was studied, in part, by a single-gene approach and by low-density microarray (Buchstaller et al. 2004). Here instead, we used LCM and a high-density oligo-microarray to characterize NCCs. Furthermore, it has been shown that mouse embryonic stem cells are more resistant to DNA damage than differentiated cells indicating that DNA surveillance by DNA repair genes decreases during cell maturation (Saretzki et al. 2004).

With the identification of molecular pathways involved in tumorigenesis, novel models of human carcinogenesis have been developed to further our understanding, evaluate potential therapies, and ultimately impact on patient outcome. Mouse models have significantly contributed to defining the molecular events underlying many human cancers. The mouse was therefore a useful mode to study the temporal expression of DNA repair genes during embryonic development and together with microarray technology, enabled the identification of differentially expressed genes belonging to specific DNA repair pathways and biological processes.

We discovered 11 DNA repair genes that were most highly expressed at pre-migratory E8.5 in enriched NCCs. The expression of 6 out of 11 genes has not been previously reported in the context of mouse embryology. Most of the 11 genes decreased drastically in expression in the AM of adult P90 stage mice compared to NCCs collected at post-migratory E13.5. For this reason, we believe that the correct functioning of DNA repair genes in the early stages of embryonic development is essential for the DNA damage response and for subsequent embryo formation. Our study indicates that the DNA repair machinery is predominantly active in the early phase of embryogenesis and its failure, during this time, may play a crucial role in the initial steps of carcinogenesis.

\section{Results}

\section{Neural crest cell identification and isolation}

NCCs were analyzed in the trunk portion of mouse embryos between somite 10 and 28 at E8.5 (Fig. 1A (i)), at E13.5 (Fig. 1B 
(i)) and in the adrenal gland (AG) of an adult mouse at P90 (Fig. 1C (i)). The anatomical sites of transverse sections for the collection of NCCs are denoted by horizontal yellow bars in Fig. 1, panels A (i), B (i) and C (i). The external cortical zone and the AM cells of the $A G$ in the adult mouse were clearly resolvable using an optical microscope (Fig. $1 \mathrm{C}$ (ii)). LCM was used to precisely isolate a sufficient amount of enriched populations of pre-migratory NCCs (E8.5), post-migratory NCC-derived cells (E13.5) and adult AM cells (P90). Figs. 1A (ii) and $1 \mathrm{~A}$ (iii) show pre-migratory NCCs in the dorsal part of the NT at E8.5 before and after laser cutting. Post-migratory NCCs were observed in the DRG at E13.5 after NT closure (Fig. 1B (ii)) and they were isolated by LCM in the dorsal portion of mouse embryos (Fig. 1B (ii) and Fig. 1B (iii)). Differentiated AM cells were captured by cutting the inner part of the mouse adrenal gland at P90 (Fig. 1C (ii) and Fig. 1C (iii)). To date the combined use of LCM technology and microarray analysis has not been reported in relation to the analysis of mouse premigratory NCCs at E8.5, at post-migratory E13.5 and in the AM at P90.

\section{Over-expression of neural crest cell markers}

We found 8 genes (Nes, Neurog2, Pax3, Prom1, Sox9, Twist1, Zic1 and Zic5), coding for markers of enriched pre-migratory NCCs at E8.5, of post-migratory NCC-derived cells at E13.5 and of adult AM cells at P90, whose expression was dramatically reduced during the three different stages examined (Fig. 2).

\section{Neural crest cell gene expression profiling analysis}

Gene expression profiles of microdissected enriched premigratory NCCs at E8.5 and post-migratory E13.5 NCCs during embryonic development and NCC-derived cells in adult P90 AM tissue were obtained by using high-density oligonucleotide microarrays. Analysis of the gene expression data was performed using SAM (Significance Analysis of Microarrays; see Supplementary Table 1). We observed that the number of genes differentially expressed between pre-migratory E8.5 and post-migratory E13.5 was higher than that expressed between post-migratory E13.5 and P90 adult AM stage; a total of 498 genes were found to be differentially expressed between pre-migratory E8.5

A

E8.5

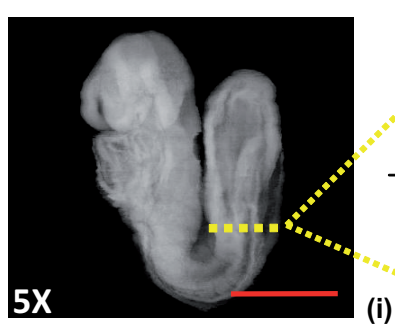

(i)

B
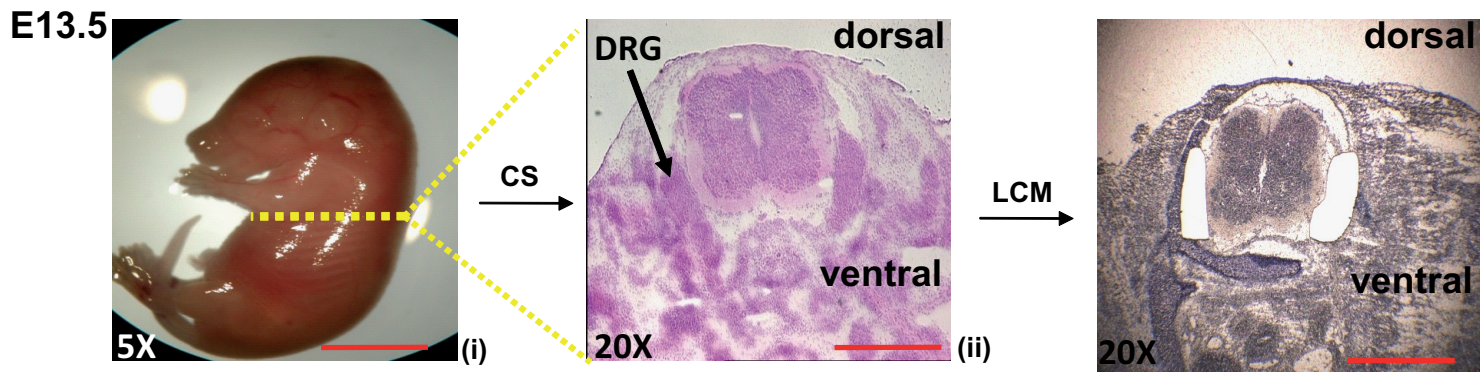

(iii)

C

\section{$\mathrm{P} 90$}

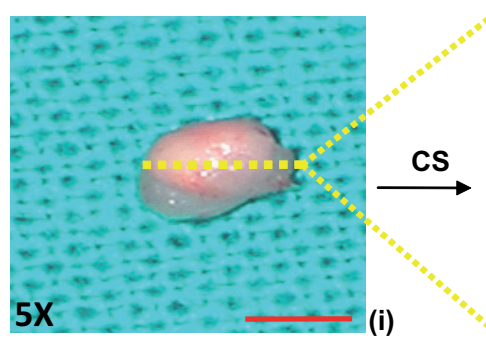

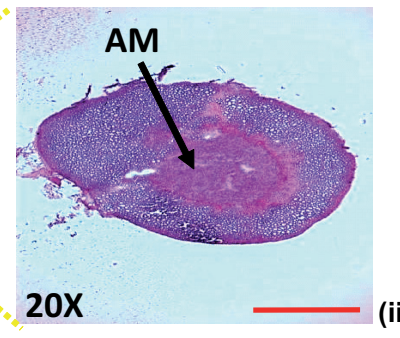

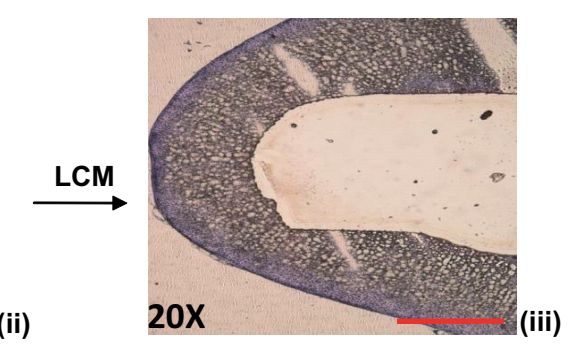

Fig.1. Identification and isolation of murine pre-, post-migratory and adult differentiated neural crest cells (NCCs). (A,B,C) Embryos at E8.5, at E13.5 and the adrenal gland in a 3 month old mouse at P90, respectively. Images (i) show whole mouse embryos and the adrenal gland with the sites of cryosectioning denoted by horizontal yellow lines. CS, cryosection. Images (ii) are H\&E stained (detailed) pictures in which black arrows indicate: trunk NCCS that were detectable in the dorsal portion of the neural tube (NT), sensory neurons in the dorsal root ganglion (DRG) and endocrine cells in the adrenal medulla (AM). Sequential isolation of enriched cellular populations was performed and in images (iii), sections that have undergone

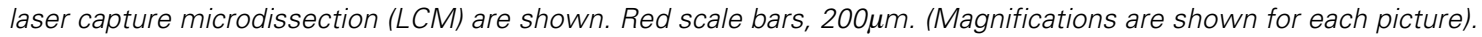


and post-migratory E13.5, whereas 176 genes were differentially expressed between E13.5 and P90 (Table 1 panel A). All genes differentially expressed (q-value $(\%)=0$ ) between the two developmental stages and the adult stage were grouped into functional categories and loaded into the EASE database, (http:// david.niaid.nih.gov/david/ease.htm) (Douglas et al. 2003) in order to identify over represented gene categories (EASE score $\geq$ 0.05). Only Gene Ontology (GO) Biological Process results from EASE were considered for our enriched pre-migratory NCCs and post-migratory NCC-derived cells analyses (Table 1 panel $B$ and panel C).

\section{DNA repair gene over-expression}

Our gene expression data showed that 11 DNA repair genes (Apex1, Chaf1b, Chek1, Clspn, Eme1, Ercc6l, Exo1, Rad18, Recc1, Ris2 and Uhrf1) were over-expressed in enriched premigratory NCCs at E8.5 and that the expression of these genes decreases remarkably in adult P90 stage in the AM compared to NCCs collected at post-migratory E13.5 (Fig. 3). Moreover, six genes (Chek1, Clspn, Eme1, Exo1, Recc1 and Uhrf1) have not been previously reported as over-expressed in mouse embryos. Chaf1b, Clspn, Ercc6l, Recc1and Ris2are involved in Nucleotide Excision Repair (NER), Apex1and Uhrf1 in Base Excision Repair (BER), Eme1 in Homologous Recombination (HR), Exo1 in Mismatch Repair (MMR), Chek1 in DNA damage response and Rad18 in the Rad6 pathway (Table 2).

We validated the expression of the 11 DNA repair genes by quantitative real-time PCR (QRT-PCR) that confirmed the temporal trend revealed by the transcriptome analysis results (Table 2). Notably, only Rad18 and Ris2 maintained constant expression

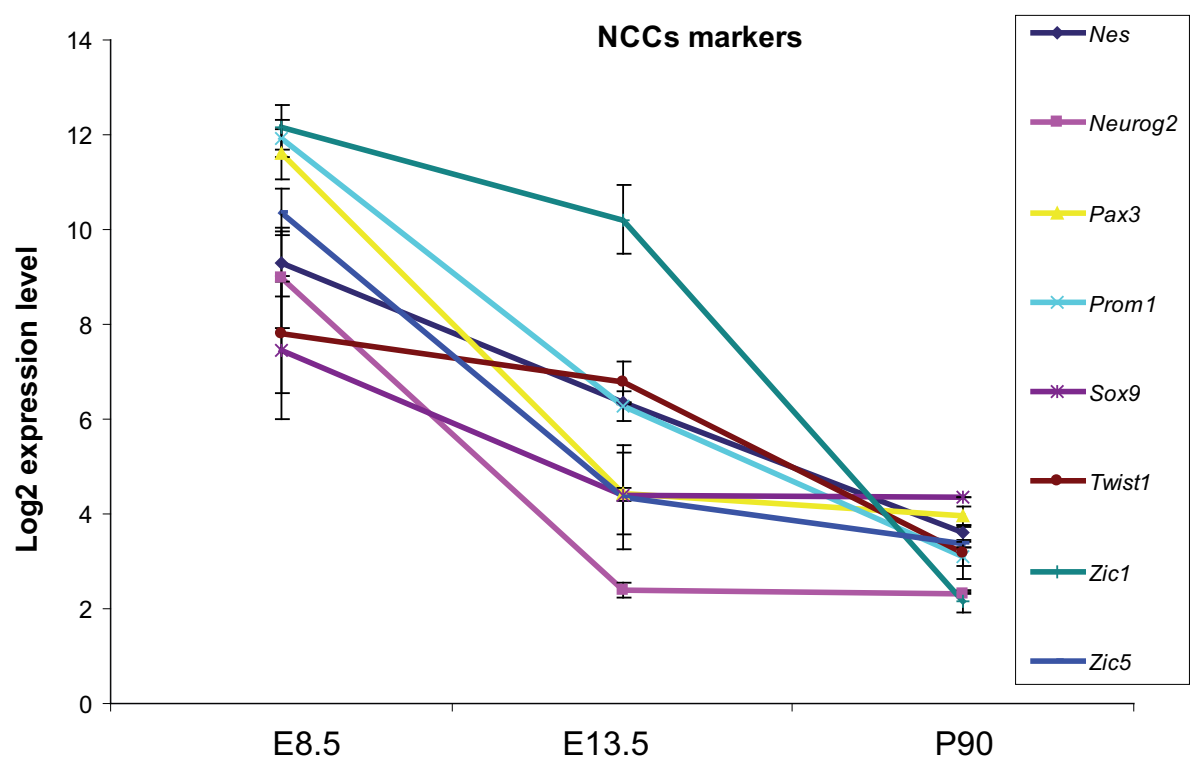

Fig. 2. Expression profiles of 8 neural crest cell (NCC) marker genes during mouse embryo developmental stages (E8.5 and E13.5), and adult (P90) stage. The graph shows line plots of each of 8 NCC markers; data were obtained from SAM analysis. On the y axis is shown the log2 expression value and on the $x$ axis is shown the mouse stages analyzed. Error bars indicate standard deviation. Standard deviation was calculated for each triplicate expression value for all 8 genes. NCCs markers dramatically reduced their expression along the three different stages examined: from pre-migratory E8.5 to post-migratory E13.5 to adult P90 AM. Notably, only Sox9 and Neurog2 genes maintained constant expression values from E13.5 to P90.
TABLE 2

\section{QRT-PCR EXPERIMENTS FOR 11 DNA REPAIR GENES}

\begin{tabular}{llccc} 
Gene & DNA repair activity/pathway & E8.5 & E13.5 & P90 \\
\hline Apex1 & response to DNA damage stimulus/BER & 5.43 & 3.63 & 3.19 \\
Chaf1b & response to DNA damage stimulus/NER & 5.36 & 2.86 & 1.10 \\
Chek1 & DNA damage checkpoint/damage response & 10.1 & 3.28 & 1.69 \\
Clspn & DNA replication checkpoint/NER & 4.37 & 2.56 & 1.25 \\
Eme1 & response to DNA damage stimulus/HR & 4.79 & 2.7 & 1.57 \\
Ercc6l & excision repair/NER & 5.63 & 2.07 & 0.65 \\
Exo1 & mismatch repair/MMR & 9.29 & 2.21 & 0.28 \\
Rad18 & response to DNA damage stimulus/Rad6 pathway & 3.83 & 3.34 & 3.08 \\
Recc1 & DNA replication/NER & 13.3 & 5.35 & 3.75 \\
Ris2 & DNA replication checkpoint/NER & 0.69 & 0.59 & 0.50 \\
Uhrf1 & DNA maintenance/BER & 3.67 & 0.78 & 0.08 \\
\hline
\end{tabular}

Table shows: gene name in alphabetical order, gene function in DNA repair mechanisms and pathways, the average expression level in each analyzed stage.

values in enriched NCCs from post-migratory E13.5 stage compared to adult NCC-derived cells at P90 in adult AM.

\section{Neural crest cell pathway analysis}

A large number of genes was found to be over-expressed in pre-migratory E8.5 NCCs compared to post-migratory E13.5 and adult P90 AM cells, showing that during embryonic development global gene expression in NCCs is generally higher than in late stages of development. Pathway analysis on differentially expressed genes showed the following: in pre-migratory E8.5 stage, 3 genes involved in apoptosis, 13 involved in the cell cycle, DNA replication and $\mathrm{G} 1$ to $S$ transition, 11 genes involved in the mRNA processing pathway, 5 genes involved in the ubiquitin pathway; in post-migratory E13.5 stage, 9 genes involved in apoptosis, 2 genes involved in the cell cycle, DNA replication and $\mathrm{G} 1$ to $S$ transition pathways, 6 genes involved in mRNA processing pathway, 3 genes involved in the ubiquitin pathway; in adult P90 AM stage: 3 genes involved in apoptosis, only one gene involved in the mRNA processing pathway and no genes involved in the cell cycle, DNA replication or the ubiquitin pathway.

\section{Discussion}

To determine differentially expressed DNA repair genes in pre-migratory and postmigratory trunk NCCs during embryonic mouse development and in postnatal life, we performed gene expression profiling of specific murine tissues. LCM and microarray analysis were combined for the analysis of enriched NCC populations at pre- and postmigratory stages E8.5 and E13.5, respectively, and in adult AM cells at P90. To our knowledge this is the first application of LCM to isolate enriched populations of trunk NCCs in pre and post-migratory stages of the mouse embryo and in differentiated AM cells for use in gene expression profiling studies.

The temporal gene expression level of all 
8 neural crest cell markers (Nes, Neurog2, Pax3, Prom1, Sox9, Twist1, Zic1 and Zic5), was in agreement with their already wellknown physiological expression in the mouse. In fact, we observed a general decrease in gene expression levels at successive stages of development, from the early mouse embryo to the adult mouse (Fig. 2). In particular, we found two transcription factors, Neurog2 and Sox9, whose expression levels remained low and unaltered in enriched NCCs at E13.5 compared to NCCderived cells in adult P90 cells (fuchsia and violet lines, respectively, in Fig. 2). Neurog2 is a key determinant of a dynamic network that is involved in the transcriptional control of embryonic stem cell self-renewal (Sansom et al. 2009), whilst Sox9 with other Sox proteins, regulates NC development and is required for mouse adrenal gland development (Reiprich et al. 2008). We can therefore speculate that the low but constant gene expression in post-migratory E13.5 stage and in adult P90 AM cells of Neurog2 and $\operatorname{Sox} 9$ is a consequence of their function in normal NC development.

The decreasing gene expression levels of 8 neural crest cell markers during embryonic development and in adult stage, confirmed that we were efficient in isolating an enriched population of pre and post-migratory NCCs in mouse embryos and of differentiated NCC-derived cells in adult mouse. Our results relating to NCCs markers are in agreement with those of Buchstaller et al. (2004), who used a different approach to screen a small population of NCCs in early and late stages of transgenic mouse development.

Analysis of the microarray data using SAM analysis (see Supplementary Table 1) showed a total of 498 genes to be differentially expressed between pre-migratory E8.5 and postmigratory $\mathrm{E} 13.5$, whereas 176 genes were differentially expressed

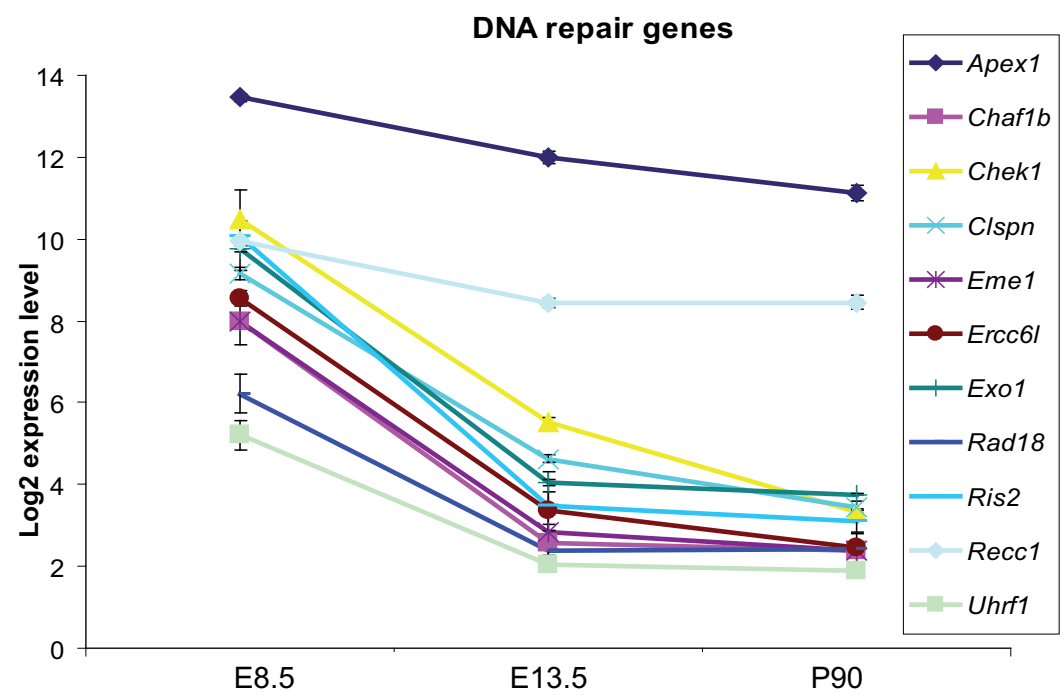

Fig. 3. Expression profiles of 11 DNA repair genes during mouse embryo developmental stages (E8.5 and E13.5), and adult (P90) stage. The graph shows line plots of each of 11 DNA repair genes obtained from SAM analysis. The y axis shows the log2 expression value and the $x$ axis shows the analyzed mouse stage. Error bars indicate standard deviation. Standard deviation was calculated for each triplicate expression value for all 11 genes. All genes showed a decreasing gene expression level from pre-migratory E8.5 to post-migratory E13.5 to adult P90 AM stage. Rad18 and Ris2 genes maintained constant expression values in NCCs from post-migratory E13.5 stage to P90 adult AM. between E13.5 and P90 (Table 1 panel A). The different gene numbers found by SAM analysis suggest that NCCs of early embryonic stages (E8.5) are highly enriched for genes involved in molecular and cellular processes compared to those of later embryonic stages (E13.5) and of adult stage (P90). Based on these results, we can argue that most NCCs at E8.5 are involved in cellular processes like cell duplication and cell proliferation that occur normally during NC development.

GO analysis was then performed in order to find classes of genes normally expressed during mouse embryonic development and in adult life stages. Table $1 \mathrm{~B}$ shows that genes upregulated in the E8.5 versus E13.5 comparison fell in gene categories (that reached an EASE score higher than 0.05), belonging to DNA replication, cell proliferation, cell cycle and cell growth and/or maintenance mechanisms. In down-regulated gene categories, there were genes belong to cell adhesion, cell communication and cell-to-cell signaling. From these observations, we can postulate that NCCs in E8.5 stage are more prone to proliferation, replication and growth rather than communication and signaling with each other. Conversely, Table $1 \mathrm{C}$ shows that there are up-regulated genes in the $\mathrm{E} 13.5$ versus $\mathrm{P} 90$ comparison and that these genes are involved in development, neurogenesis and organogenesis. This could reflect the organ formation processes in which E13.5 NCCs are involved during murine development. Down-regulated gene categories for this comparison belong to steroid, hormone and lipid biosynthesis pathways thus verifying that AM cells isolated by LCM were enriched of typical chromaffin cells that secrete adrenaline, noradrenaline and endogenous ligands or specifically, endorphins.

Pathway analysis results obtained from enriched pre-migraory NCCs, post-migratory NCC-derived cells and P90 adult AM cells overlap the results reported by Wang etal. (2004), which demonstrated fetal-like neural progenitors are more susceptible to cancer initiation due to the deregulation of specific pathways.

Gene expression data showed that 11 DNA repair genes (Apex1, Chaf1b, Chek1, Clspn, Eme1, Ercc6l, Ex01, Rad18, Recc1, Ris2 and Uhrf1) were overexpressed in enriched pre-migratory NCCs at E8.5 and that their expression decreased markedly at postmigratory E13.5, and even further in P90 adult AM (Fig. 3). Notably, DNA repair pathways in which the 11 overexpressed genes are involved are as follows: Nucleotide Excision Repair (NER): Chaf1b, Clspn, Ercc6l, Recc1 and Ris2, Base Excision Repair (BER): Apex1 and Uhrf1; Homologous Recombination (HR): Eme1; Mismatch Repair (MMR): Exo1; DNA damage response: Chek1, and in the Rad6 pathway, Rad18. Taken together our results indicate that pre-migratory NCCs at early E8.5 stage are likely to use NER for DNA repair even ifÄavailable data from conflicting reports (Tichy and Stambrook, 2008; Maynard et al. 2008) do not fully clarify the dominant mechanism of DNA repair in premigratory NCCs versus post-migratory and differentiated NCCs.

Noteworthy, Rad18 and Ris2 genes maintained constant expression levels at E13.5 and at P90 stages (Fig. 3, dark blue and turquoise lines, respectively).

Rad18 has been shown to be involved in transmit- 
ting the damage response signal to orchestrate homologous recombination repair (HRR) (Huang et al. 2009) and, moreover, it contributes to the maintenance of genomic stability through post-replication repair in $R A D 18$ knockout mouse embryonic stem cells (Tateishi et al. 2003).

The Ris2 gene (also called Cdt 1 ) is probably the most important target for DNA damage in higher eukaryotes (Tada, 2007). Its mechanism of action has been described by Spella et al. (2007). Cdt1 is abundantly expressed by neural progenitor cells during early mouse neurogenesis and its expression level declines at late developmental stages becoming undetectable upon differentiation.

We can therefore hypothesize that the constant gene expression of Rad18and Ris2DNA repair genes in post-migratory NCCs at E13.5 and at P90 in adult AM cells is critical for maintaining the genomic stability of NCCs. The constant gene expression values observed for Rad18and Ris2 in post-migratory E13.5 and P90 adult AM cells can explain why we did not find them differentially expressed in SAM analysis.

One interesting DNA repair gene observed as differentially expressed in our microarray analysis of NCCs model is Apex 1 (Apurinic/apyrimidinic (AP) multifunctional DNA repair enzyme 1). Apex 1 has the highest expression level of the 11 DNA repair genes (blue line with rhombus in top part of Fig. 3), and belongs to the BER pathway. Concerning this gene, in their review, Vinson and Hales (2002), discussed several papers reporting the over-expression of Apex 1 in mouse embryonic NCCs. In fact, Vinson and Hales summarized the up-regulation of several DNA repair genes during mouse development as critical steps during mammalian organogenesis. In addition, two articles (Ono et al. 1995; Wilson et al. 1996) have shown that Apex 1 is one of the DNA repair genes whose expression is temporally regulated during the lifetime of an organism. Indeed, the major conclusions of these two above reports were that Apex1 stimulates the DNA binding activity of numerous transcription factors that are involved in cancer promotion and progression such as: AP-1 (Fos/Jun), NFÍB, HIF-1 $\alpha$, CREB and p53 (Fishel and Kelley, 2007). This result suggests that Apex 1 is a candidate gene involved in tumor initiation.

Furthermore, Saretzki et al. (2004) reported that expression of some DNA repair genes was down-regulated during the early stages of differentiation of murine embryonic stem cells by using microarrays and reverse transcription-polymerase chain reaction (RT-PCR). These authors identified several genes involved in cellular responses to stress and in DNA repair pathways that were down-regulated during the differentiation of ES cells. Amongst the 11 DNA repair genes that we observed as over-expressed in early embryonic stages, Recc1 had the third highest expression (light blue line in Fig. 3). Recc1 has been described as a DNA polymerase accessory protein required for the coordinated synthesis of both DNA strands during SV40 DNA replication in vitro(Uchiumi et al. 1996). It was found that the large subunit of Recc1 binds to DNA sequence repeats of telomeres. Finally, the down-regulation of Recc 1 during NCC differentiation found here is in agreement with the observations of 3 other investigators (Buchstaller J, 2004; Hiyama E, 1995; Schmitt $\mathrm{H}, 1994)$. This suggests that the deregulation of Recc1 in NCCs can contribute to cancer initiation.

Eme1, another down-regulated gene, plays a key role in
DNA repair and the maintenance of genome integrity in mammalian cells (Abraham et al. 2003), supporting our result on the high expression in pre-migratory NCCs at E8.5 and in postmigratory NCCs at E13.5 with subsequent decreasing expression at $\mathrm{P} 90$ in adult $\mathrm{AM}$ cells.

Uhrf1, also known as NP95 in mouse and ICBP90 in human, was shown to be almost completely colocalized with chromatinbound PCNA throughout the nuclei in early S phase and also partly in mid-S phase (Muto et al. 2002). Furthermore, Bostick et al. (2007), demonstrated that NP95 functions as a common component of multiple response pathways for DNA damage and that this gene plays some role in the maintenance of genomic stability.

Our results therefore provide novel and supportive data relating to previous observations (Vinson and Hales, 2002; Buchstaller et al. 2004), of a temporal down-regulation of DNA repair genes during mouse development and in adult life. Thus, the over-expression of DNA repair genes during the early phases of embryonic life strongly support the hypothesis that this class of genes contributes to the genomic stability and maintenance system of somatic cells. In fact, premature decreases in the expression of DNA repair genes may result in increased DNA damage and consequently cancer pathogenesis. With respect to this, Kennedy and D'Andrea (2006) reported that pediatric cancer susceptibility syndromes are strictly associated with the failure of DNA repair genes. It is therefore possible that the deregulation of DNA repair genes initiates carcinogenesis.

In conclusion, we discovered 11 DNA repair genes to be the most highly expressed in pre-migratory E8.5 enriched NCCs. The expression of 6 of these 11 genes has not been previously reported in mouse embryology. Furthermore, most of the 11 genes decreased drastically in expression of adult P90 (in the AM), compared to NCCs collected at post-migratory E13.5. For this reason, we believe that a correct activity of DNA repair genes in the early stages of embryo development is an essential condition to ensure the physiological process of DNA damage response and embryonic formation. Moreover, because different reports have shown that early NCCs have a higher capacity to repair damaged DNA compared to differentiated cells, these data collectively suggest that transcriptional profiling of pre- and post-migratory NCCs needs to be further refined in order to permit researchers to better understand the role of DNA repair genes in the biology of cancer pathogenesis steps. We therefore strongly recommend the use of enriched pre-migratory NCCs and post-migratory NCC-derived cells as models to investigate the key roles of DNA repair genes in cancers with an underlying NCC-related pathogenesis for gene therapy development. Moreover, as the use of microdissected enriched pre-migratory NCCs and post-migratory NCC-derived cells allowed us to identify selected DNA repair genes at the transcriptional level, we believe that this model could be used to perform functional studies of preferred embryonic and adult tissue cells.

Finally, we report here the first gene expression profiling analysis of E8.5 and E13.5 mouse embryonic NCCs and P90 AM-derived cells using LCM and high-density oligo-microarray. From the present work we have generated a NCCs and NCCderived cells gene expression database that can be utilized by 
the scientific community to further investigate the role of gene(s) involved in embryonic development mechanisms.

In vitro and in vivo functional studies currently being performed in our laboratory to understand better the roles of the 11 DNA repair genes discussed here in relation to mouse development and to the initiation of carcinogenesis.

\section{Materials and Methods}

\section{Animal model and preparation of cryosections for staining}

We used outbred CD-1 pregnant female mice and the day of appearance of the vaginal plug was considered as E0.5. Mice were sacrificed by cervical dislocation and the embryos were quickly separated from the placenta and chilled in cold D-PBS1X (w/o Ca/Mg) (Cambrex Bio Science, Belgium). The embryos were then transferred to cold sucrose $(30 \% \mathrm{v} / \mathrm{v}$ in DEPC-treated water (Invitrogen, Carlsbad, CA)), and incubated for 30 minutes at $4^{\circ} \mathrm{C}$. Following this, the embryos were embedded in OCT (Tissue-Tek OCT \#4583, Sakura Finetek USA), frozen in dry ice and stored at $-80^{\circ} \mathrm{C}$. Embryonic sections of 4 to $6 \mu \mathrm{m}$ were cut at $-20^{\circ} \mathrm{C}$ using the Leica CM1100 cryostat (Leica Microsystem, Bannockburn, IL, USA). Each sample was stained with Haematoxylin \& Eosin (H\&E) to verify its integrity and to enable microscopic assessment of cell morphology.

\section{Laser capture microdissection (LCM) experiments and RNA isola- tion of NCCs}

LCM was used to isolate enriched populations of NCCs at premigratory E8.5 and post migratory E13.5, as well as P90 NCC-derived cells from the adult AM (AS LMD Microsystem, Leica Microsystem). The system consists of a computer-assisted laser microscope that can be used to safely isolate a few cells from frozen tissue sections. Prior to microdissection, cells were fixed and stained with $\mathrm{H} \& \mathrm{E}$ according to the manufacturer's instructions. Transverse frozen sections of $4 \mu \mathrm{m}$ were cut and mounted onto PEN (polyethylene naphthalate) membrane slides from Leica, using a CM-1100 cryostat (Leica Microsystems).

We microdissected and isolated 500-700 NCCs at pre-migratory E8.5; 2,000 DRG cells at post-migratory E13.5 and 2,500-3,000 AM cells from adult mice (P90). Staining and microdissection were performed rapidly to maintain RNA integrity. Total RNA was immediately purified using the PicoPure ${ }^{\mathrm{TM}} \mathrm{RNA}$ isolation kit (Arcturus Engineering, Mountain View, CA). A volume of extraction buffer ranging from $12 \mu \mathrm{l}$ to $16 \mu \mathrm{l}$ was used for each tube, depending on the number of captured cells. Total RNA was analysed with a 2100 BioAnalyzer instrument (Agilent Technologies $\mathrm{GmbH}$, Waldbronn, Germany), and only RNA samples with an RNA integrity number $\geq 7$ were included in the study.

Production of antisense RNA, synthesis of biotin-labeled cRNA and GeneChip hybridization

Total RNA was linearly amplified using the GeneChip ${ }^{\circledR}$ Eukaryotic Small Sample Target labeling Assay Version II (Affymetrix, Inc., CA, USA) to obtain a sufficient amount of cRNA for microarray hybridization. We performed 3"biological replicates for each embryonic stage, and adult tissue. Genome-wide expression analysis was performed by using the Mouse Genome 4302.0 GeneChip $\circledast$ (Affymetrix). The manufacture's instructions were followed for microarray analysis.

\section{Gene expression profiling data analysis}

CEL files obtained from the GeneChip Operating Software (GCOS, Affymetrix), were analyzed using the Significance Analysis of Microarrays (SAM) approach (Tusher et al. 2001). Data were normalized according to the GeneChip robust multi-array average (GCRMA) algorithm of the R/ Bioconductor 1.4 package (http://www.bioconductor.org). Genes with statistically significant expression changes were determined using permutation tests SAM (http://www-stat.stanford.edu/ tibs/SAM/). Genes altered by at least two-fold between conditions were considered. The $\Delta$ value was set to return a median false significant number $=0$. The $q$ values were 0 for the different gene lists created. Annotations were obtained through the Database for Annotation, Visualization, and Integrated Discovery (DAVID; http://david.niaid.nih.gov/david/beta/index.htm) (Dennis et al. 2003). Standard deviation was calculated for each of all 8 NCCs markers and for each of all 11 DNA repair genes by using the expression value average in triplicate experiments.

\section{Quantitative real-time PCR (QRT-PCR)}

Total RNA obtained from the LCM-isolated NCCs was retro-transcribed using random hexamers and Superscript II reverse transcriptase (Invitrogen Life Technologies Inc., Carlsbad, CA). QRT-PCR analysis was performed on an EpGradient Realplex ${ }^{4}$ PCR System (Eppendorf AG, Hamburg, Germany) using the RealMaster Mix Probe (Eppendorf AG) according to the manufacturer's protocol. FAM-labeled TaqMan assays for the 11 DNA repair genes and the glyceraldehyde-3-phosphate dehydrogenase (Gapdh) endogenous control were designed by Primer Design Ltd (Hants, UK). The comparative $\mathrm{C}_{\mathrm{T}}$ method was used in order to evaluate the differential DNA repair gene expression in each NCCs stage with respect to a common calibrator sample (mouse embryo BioBank cDNA, Primer Design Ltd). The relative amount of each target gene to Gapdh was determined using the following equation:

$2^{-\Delta \Delta \mathrm{Ct}}$, where $\Delta \Delta \mathrm{Ct}_{\text {gene }}=\left[\mathrm{Ct}_{\text {gene }}-\mathrm{Ct}_{\text {Gapdh }}\right]_{\mathrm{NCCs}}-\left[\mathrm{Ct}_{\text {gene }}-\mathrm{Ct}_{\text {Gapdh }}\right]_{\text {calibrator }}$. For each target gene we performed three replicates of QRT-PCR.

\section{Acknowledgements}

We are very grateful to Afua Mensah (Oncology Institute of Southern Switzerland (IOSI), Laboratory of Experimental Oncology, Bellinzona, CH) for manuscript reading, comments and suggestions. This work was supported by: Italian Neuroblastoma Foundation, Ministero dell'struzione, dell'Università e della Ricerca (FIRB), Ministero della Salute, EU project NewGeneris, EU 6FP (FOOD-CT-2005-016320). D.A. is a fellow of the Italian Neuroblastoma Foundation.

\section{References}

ABRAHAM J, LEMMERS B, HANDE MP, MOYNAHAN ME, CHAHWAN C, CICCIA A, ESSERS J, HANADA K, CHAHWAN R, KHAW AK, MCPHERSON P, SHEHABELDIN A, LAISTER R, ARROWSMITH C, KANAAR R, WEST SC, JASIN M, HAKEM R (2003). Eme1 is involved in DNA damage processing and maintenance of genomic stability in mammalian cells. EMBO J22: 6137-6147.

BOSTICK M, KIM JK, ESTÈVE PO, CLARK A, PRADHAN S, JACOBSEN SE (2007). UHRF1 plays a role in maintaining DNA methylation in mammalian cells. Science 317: 1760-1764.

BUCHSTALLER J, SOMMER L, BODMER M, HOFFMANN R, SUTER U, MANTEI N (2004). Efficient Isolation and Gene Expression Profiling of Small Numbers of Neural Crest Stem Cells and Developing Schwann Cells. J Neurosci24: 23572365.

DENNIS GJR, SHERMAN BT, HOSACK DA, YANG J, GAO W, LANE HC LEMPICKI R (2003). DAVID: Database for annotation, visualization, and integrated discovery. Genome Bio/4: P3.

DOUGLAS AH, GLYNN DJR, BRAD TS, CLIFFORD LH, RICHARD AL (2003). Identifying Biological Themes within Lists of Genes with EASE. Genome Biology 4: P4.

DUSRE L, COVEY JM, COLLINS C, SINKA BK (1989). DNA damage, cytotoxicity and free radical formation by mitomycin $\mathrm{C}$ in human cells. Chem Biol Interact 71 : 63-78.

FALCHETTI ML, LAROCCA LM, PALLINI R (2002). Telomerase in brain tumors. Childs Nerv Syst 18: 112-117.

FISHEL ML, KELLEY MR (2007). The DNA base excision repair protein Ape1/Ref1 as a therapeutic and chemopreventive target. Mol Aspects Med28: 375-395.

FROSINA G (2000). Overexpression of enzymes that repair endogenous damage to DNA. Eur J Biochem 267: 2135-2149.

GAMMILL LS, BRONNER-FRASER M (2003). Neural crest specification: migrating into genomics. Nat Rev Neurosci 4: 795-805. 


\section{D. Albino et al.}

HERGENHAHN M, KENZELMANN M, GRÖNE HJ (2003). Laser controlled microdissection of tissues opens a window of new opportunities. Pathol Res Pract 199: 419423.

HIYAMA E, HIYAMA K, YOKOYAMA T, MATSUURA Y, PIATYSZEK MA, SHAY JW (1995). Correlating telomerase activity levels with human neuroblastoma outcomes. Nat Med 1: 249-255.

HUANG J, HUEN MS, KIM H, LEUNG CC, GLOVER JN, YU X, CHEN J (2009). RAD18 transmits DNA damage signalling to elicit homologous recombination repair. Nat Cell Bio 11: 592-603.

JAROUDI S, SENGUPTA S (2007). DNA repair in mammalian embryos. Mutation Research 635: 53-77.

KENNEDY RD, D'ANDREA AD (2006). DNA repair pathways in clinical practice: lessons from pediatric cancer susceptibility syndromes. J Clin Onco/24: 37993808.

LE DOUARIN NM, KALCHEIM C (Ed 2) (1999). The Neural Crest. Cambridge, UK: Cambridge University Press: p. 445.

MACDONALD TJ (2008). Aggressive infantile embryonal tumors. JChildNeuro/23: 1195-1204.

MAYNARD S, SWISTOWSKA AM, LEE JW, LIU Y, LIU ST, DA CRUZ AB, RAO M, DE SOUZA-PINTO NC, ZENG X, BOHR VA (2008). Human embryonic stem cells have enhanced repair of multiple forms of DNA damage. Stem Cells 26: 2266-2274.

MUTO M, KANARI Y, KUBO E, TAKABE T, KURIHARA T, FUJIMORI A, TATSUMI $K$ (2002). Targeted disruption of Np95 gene renders murine embryonic stem cells hypersensitive to DNA damaging agents and DNA replication blocks. J Bio/ Chem 277: 34549-34555.

OHALI A, AVIGAD S, ASH S, GOSHEN Y, LURIA D, FEINMESSER M, ZAIZOV R, YANIV I (2006). Telomere length is a prognostic factor in neuroblastoma. Cancer107: 1391-1399.

ONO Y, WATANABE M, INOUE Y, OHMOTO T, AKIYAMA K, TSUTSUI K, SEKI S (1995). Developmental expression of APEX nuclease, a multifunctional DNA repair enzyme, in mouse brains. Dev Brain Res 86: 1-6.

REIPRICH S, STOLT CC, SCHREINER S, PARLATO R, WEGNER M (2008). SoxE proteins are differentially required in mouse adrenal gland development. $\mathrm{MO} /$ Biol Cel/ 19: 1575-1586.
SANSOM SN, GRIFFITHS DS, FAEDO A, KLEINJAN DJ, RUAN Y, SMITH J, VAN HEYNINGEN V, RUBENSTEIN JL, LIVESEY FJ (2009). The level of the transcription factor $P a x 6$ is essential for controlling the balance between neural stem cell self-renewal and neurogenesis. PLOS Genet5: e1000511.

SARETZKI G, ARMSTRONG L, LEAKE A, LAKO M, VON ZGLINICKI T (2004). Stress defense in murine embryonic stem cells is superior to that of various differentiated murine cells. Stem Cel/s 22: 962-971.

SCHMITT H, BLIN N, ZANKL H, SCHERTHAN H (1994). Telomere length variation in normal and malignant human tissues. Genes Chromosomes Cancer11: 171 177.

SPELLA M, BRITZ O, KOTANTAKI P, LYGEROU Z, NISHITANI H, RAMSAY RG, FLORDELLIS C, GUILLEMOT F, MANTAMADIOTIS T, TARAVIRAS S (2007). Licensing regulators Geminin and Cdt1 identify progenitor cells of the mouse CNS in a specific phase of the cell cycle. Neuroscience 147: 373-387.

TADA S (2007). Cdt1 and geminin: role during cell cycle progression and DNA damage in higher eukaryotes. Front Biosci 12: 1629-1641.

TATEISHI S, NIWA H, MIYAZAKI J, FUJIMOTO S, INOUE H, YAMAIZUMI M (2003). Enhanced genomic instability and defective postreplication repair in RAD18 knockout mouse embryonic stem cells. Mol Cell Biol23: 474-81.

TICHY ED, STAMBROOK PJ (2008). DNA repair in murine embryonic stem cells and differentiated cells. Exp Cell Res 314: 1929-1936.

TUSHER VG, TIBSHIRANI R, CHU G (2001). Significance analysis of microarrays applied to the ionizing radiation response. Proc Nat/ Acad Sci USA 98: 51165121.

UCHIUMI F, OHTA T, TANUMA S (1996). Replication factor C recognizes 5 phosphate ends of telomeres. Biochem Biophys Res Commun 229: 310-315.

VINSON RK, HALES BF (2002). DNA repair during organogenesis. Mutation Research 509: 79-91.

WANG Y, BAI Y, LI X, HU Q, LIN C, XIAO Z, LIU Y, XU J, SHEN LICA, LI LICA (2004). Fetal human neural progenitors can be the target for tumor transformation. Neuroreport 15: 1907-1912.

WILSON TM, RIVKEES SA, DEUTSCH WA, KELLEY MR (1996). Differential expression of the apurinic/apyrimidinic endonuclease (APE/ref-1) multifunctional DNA base excision repair gene during fetal development and in adult rat brain and testis. Mutat Res 362: 237-248. 


\section{Further Related Reading, published previously in the Int. J. Dev. Biol.}

See our recent Special Issue Placenta edited by Joan S. Hunt and Kent L. Thornburg at:

http://www.ijdb.ehu.es/web/contents.php?vol=54\&issue=2-3

Characterization of mouse embryonic stem cell differentiation into the pancreatic lineage in vitro by transcriptional profiling, quantitative RT-PCR and immunocytochemistry

Alexandra Rolletschek, Insa S. Schroeder, Herbert Schulz, Oliver Hummel, Norbert Huebner and Anna M. Wobus

Int. J. Dev. Biol. (2010) 54: 41-54

Neural crest ontogeny during secondary neurulation: a gene expression pattern study in the chick embryo Liliana Osório, Marie-Aimée Teillet, Isabel Palmeirim and Martin Catala

Int. J. Dev. Biol. (2009) 53: 641-648.

Transcriptional regulation by Pax3 and TGFbeta2 signaling: a potential gene regulatory network in neural crest development Hiromichi Nakazaki, Yueh-Wei Shen, Beth Yun, Anvesh Reddy,Varun Khann, Barbara Mania-Farnell, Shunsuke Ichi, David G. Mclone, Tadanori Tomita and C. Shekhar K. Mayanil*

Int. J. Dev. Biol. (2009) 53: 69-79.

Pax7 identifies neural crest, chromatophore lineages and pigment stem cells during zebrafish development Ana M Lacosta, Jesús Canudas, Cristina González, Pedro Muniesa, Manuel Sarasa and Luis Domínguez Int. J. Dev. Biol. (2007) 51: 327-331.

Restriction of the fate of early migrating trunk neural crest in gangliogenesis of avian embryos $\mathrm{K}$ Asamoto, Y Nojyo and H Aoyama Int. J. Dev. Biol. (1995) 39: 975-984.

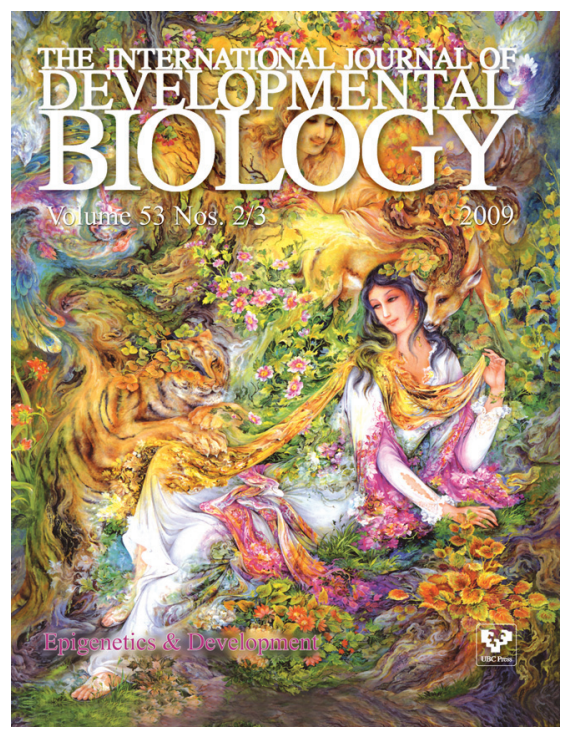

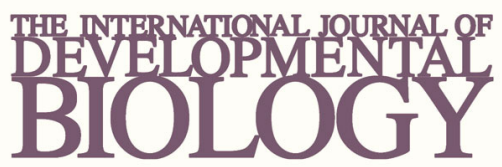

Volume 54 Nos. 6/7
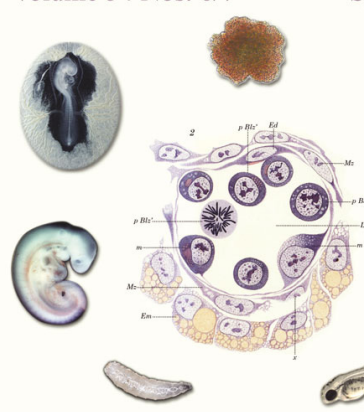

Developmental Hematopoiesis
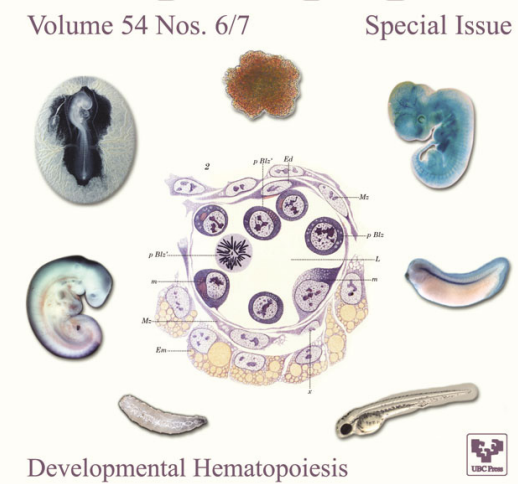

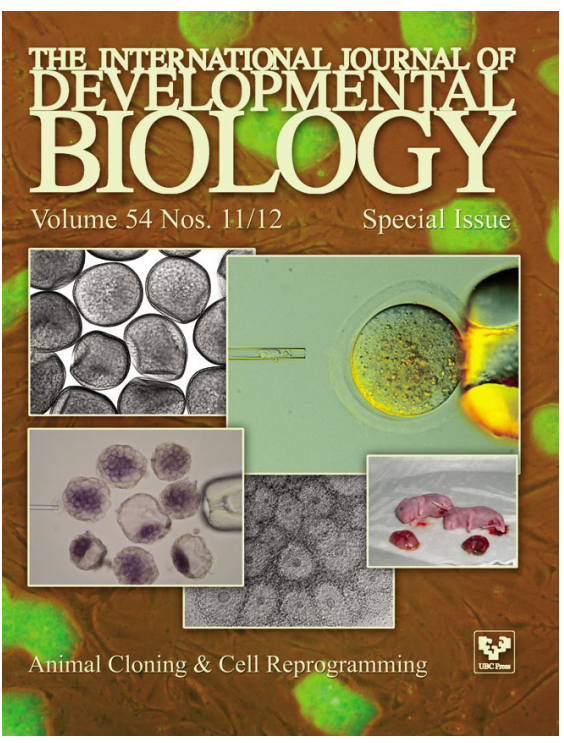

